NATASCHA UND WERNER SEIBT / WIEN

\title{
SIEGEL DER SAMMLUNG ORGHIDAN
}

\section{Eine Nachlese zur Edition V. Laurents}

Die byzantinische Siegelkunde verdankt R. P. Vitalien Laurent entscheidende Impulse für die beachtlichen Fortschritte, die sie in den letzten Jahrzehnten erreichen konnte. Der mangelhafte Erhaltungszustand bzw. die Fragmentierung der Mehrheit der auf uns gekommenen Siegel zwingt auch heute noch oft zu Hypothesen, die später bisweilen bestätigt werden, oft aber auch zu korrigieren sind.

Die Edition der Sammlung Orghidan, die sich in Bukarest befindet, legte Laurent vor einem halben Jahrhundert vor. Da wir sie durchsahen, um Material für die systematische Photothek der Kommission für Byzantinistik der Österreichischen Akademie der Wissenschaften zu gewinnen, ergaben sich - trotz des schlechten Zustandes vieler Siegel und der geringen Qualität der Abbildungen - so manche Anmerkungen, teilweise stützten neue Parallelstücke fragliche Lesungen, teilweise ermöglichten sie auch Korrekturen. Eine Auswahl soll im folgenden vorgelegt werden, vor allem um zu verhindern, dass neue Hypothesen „auf Sand gebaut“ werden.

1. Es handelt sich wohl um ein rein ikonographisches Siegel, der „empereur anonyme" ist eher als Erzengel Michael zu interpretieren.

5. Eine Zuweisung an Nikephoros Botaneiates ist sicher abzulehnen; eher wäre an Basileios II. zu denken (vgl. Zacos - Vegler $Y^{1} 75$ b und c).

6. Vielleicht Konstantin X. Dukas (vgl. Zacos - Veglery 87).

9. Die Zuweisung an Ioannes II. Komnenos überzeugt nicht.

11. Auf dem Parallelstück der Fogg-Sammlung schlagen die Editoren

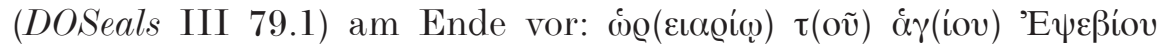
(statt Eủocßíov).

28. Ein Parallelstück in Athen (371) bestätigt die Lesung, auch wenn Konstantopulos nur einen Teil des Namens entziffern konnte.

${ }^{1}$ Für die Vollzitate der gängigen sigillographischen Abkürzungen sei z. B. auf W. SEIBT - M. L. Zarnitz, Das byzantinische Bleisiegel als Kunstwerk. Wien 1997, 212-215 verwiesen. 
38. Zacos und Veglery datieren ein Parallelstück (Nr. 1903) richtig ins 8. Jh.

41. Sicher nicht 9.-10. Jh., sondern 8., vielleicht sogar 1. Hälfte.

49. Wiederholt in Laurent, Corpus II 170 (im folgenden nur: Corpus). Am Ende ist eher - wie auf einem zumindest sehr ähnlichen Stück

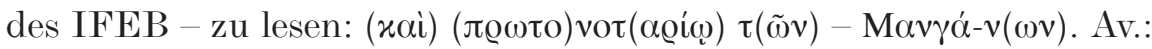
Büste der Theotokos Nikopoios oder im Minimalorantengestus.

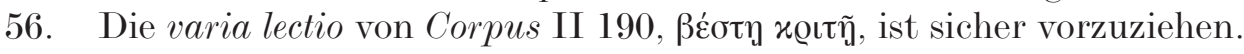
Der Mann ist ohne Zweifel mit dem von Corpus II $211 \mathrm{zu}$ verbinden (zumindest die Rv.-Seite könnte stempelidentisch sein). Vielleicht war diese Person sogar kaiserlicher Protonotar.

57. Aus Gründen der inneren Symmetrie ist ein Beta am Beginn der Rv.Legende auszuschließen.

68. Auch wenn das Siegel nicht abgebildet ist, handelt es sich wohl um

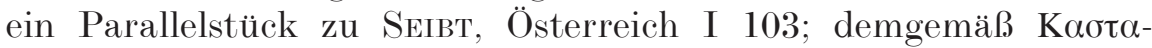

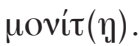

70. Ein Parallelstück in D. O. (Neg.Nr. 61.17.29-771). Das Av.-Monogramm weist rechts außen sicher Eta auf, das Tetragramm lautet ungewöhnlich TO CO $\Delta \mathrm{OV}-\Lambda \mathrm{W}$ (O-V ligiert). 2. Hälfte 9. bis Anfang 10. Jh.

74. Eine Verbindung von Protospathar und Bestarches ist sicher abzulehnen.

76. Der Familienname ist nicht Anzas sondern sicher Antiochos (Parallelstücke in der Sammlung Zacos und Seyrig 38; auf ersterem ist eindeutig ANTIOX, zu lesen).

77. In Corpus II 713 korrigiert (nicht Ioannes sondern Epiphanios).

78. Wie ein Parallelstück der Sammlung Zacos bestätigt, ist der Familienname $\Phi \mathrm{V} \Lambda \mathrm{O}-\mathrm{KA} \Lambda \mathrm{H}$ geschrieben.

80. $\mathrm{Zu}$ diesem Mann vgl. auch Corpus II 773.

86. Ein Parallelstück D.O. 58.106.3382, ed. Zacos - Veglery 1466.

88. Parallelstück Zacos - Vegler 1570.

90. Ein Parallelstück in Athen (Konstantopulos 408), ein recht ähnliches in Istanbul; sicher dieselbe Person wie Zacos - Veglery 831 (dort

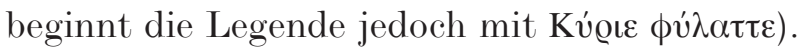

91. Es gibt mehrere Parallelstücke (Polen 23, Shaw 395 u. 1340, eines, das einst Pančenko vorlag); die Interpretation von A. Szemioth - T. Wasilewski, Commentationes 11 (1966) 20f., Nr. 23, ist der Laurents

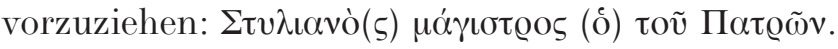

95. Wohl eher Paktiares, wie auf einem anderen Typus desselben Mannes (Seyrig 211). Siehe dort auch zur Av.-Darstellung.

96. Viele ähnliche Siegel oder echte Parallelstücke sichern die Ergänzungen ab. 


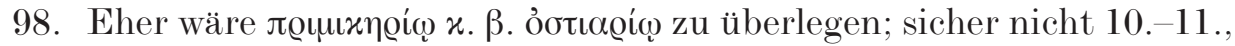
wohl Wende 9./10. Jh.

100. Die richtige Lesung des Verses auf dem - allerdings nicht stempelidentischen - Siegel Zacos II 516. Wahrscheinlich 12., nicht 13. Jh.

108. Besser bei Zacos - Vegler 455.

109. Patrikios am Ende wäre recht unwahrscheinlich.

112. Die Verbindung mit Laurent, Vatican 40 ist nicht ganz sicher. Vgl. auch SeIBT, Österreich I zu 142.

114. Zu einem Siegel desselben Bardales als Protoproedros (wobei beide Verse ,in Ordnung“ waren) s. H. W. BelL, Byzantine Sealings. BZ 30 (1929-30) 637, Nr. 12 (ein Parallelstück dazu in der Ermitaž, M-8507).

116. Außer dem Rangtitel Proedros ist vieles unsicher. Der Familienname könnte auch Skuph(as) gelautet haben.

120. Der Familienname lautet wohl $\tau \tilde{\omega}{ }^{~} A \lambda \alpha x \alpha \sigma(\sigma) \varepsilon \dot{\varepsilon}$. Parallelstück D.O. Neg.Nr. 59.109.5-2339.

123. Parallelstück D.O. Neg.Nr. 55.65.5-2510. Wende 9./10. Jh.

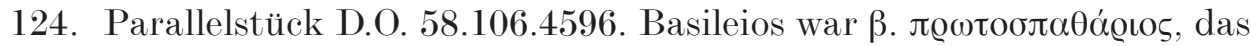

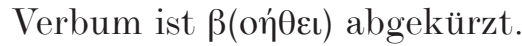

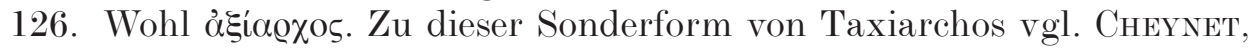
REB 44 (1986) 233-235.

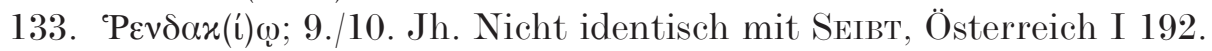

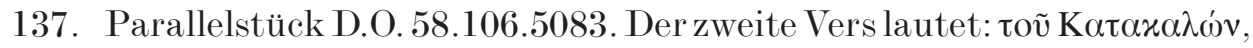

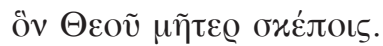

142. Vgl. jetzt Ch. Stavrakos, Die byzantinischen Bleisiegel mit Familiennamen aus der Sammlung des Numismatischen Museums Athen. Wiesbaden 2000, 46.

152. Sicher 8. Jh., ca. 2. Viertel.

154. Lesung durch gut erhaltenes Parallelstück der Sammlung Zacos bestätigt.

155. Leider nicht abgebildet; vgl. eventuell LichačEv, Vostok, Taf. LXX 8.

156. Wohl 10. Jh.

158. Familienname unglaubwürdig; vielleicht Gymnos.

160. Besser erhaltenes Parallelstück Zacos II 187.

166. S. Oikonomides, DOP 1983, 153ff. u. Zacos II 390.

168. Der Vorname ist sehr unsicher.

171. S. Seyrig 88 u. Corpus II 351.

172. Zur Datierung vgl. SeIBT, Österreich I 73.

174. Datierung im Corpus II 315 auf 9./10. Jh. korrigiert.

175. Ende unglaubwürdig. 9./10. Jh.

179. Korrigiert in Corpus II 773. 


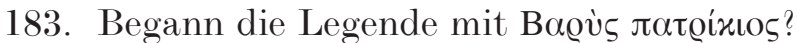

187. Wohl nur Proedros. So auch Oikonomidès, Listes 299, A. 72.

191. Die Lesung wird durch ein Parallelstück im IFEB gestützt. 2. Hälfte 10. Jh.

193. S. Seyrig 99 (am Ende ist $\tau \tilde{\omega} v$ ả $\gamma \varepsilon \lambda \tilde{\omega} v$ zu lesen).

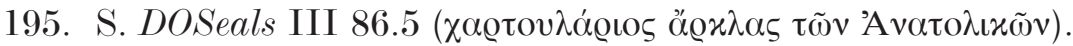

196. Vielleicht war Melissenos auf diesem Siegel doch nur Proedros.

198. Wenn Niketas nur Proedros (statt Protoproedros) war, würde die Zuweisung an den Bruder Michaels IV. an Wahrscheinlichkeit gewinnen.

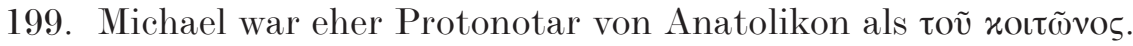

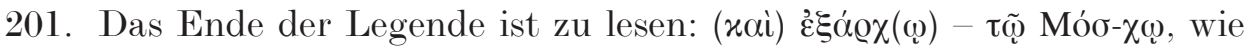
aus mehreren parallelen bzw. ähnlichen Stücken erhellt.

202. Parallelstück Zacos II 887.

205. Zu dieser Person vgl. auch Zacos - Veglery, zu Nr. 2679.

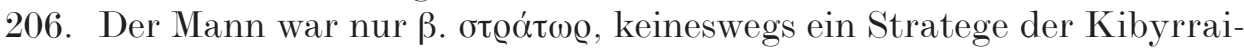
oten.

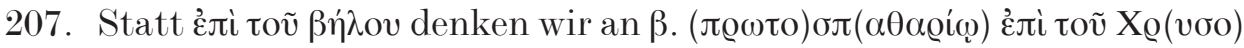

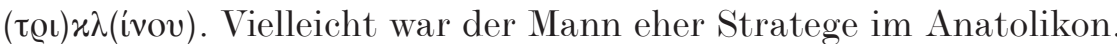

208. Vgl. jetzt auch DOSeals IV 48.3.

212. Wenn statt der X-P-Ligatur ein kleines $\mathrm{T}$ über $\mathrm{K}$ zu lesen ist, wäre

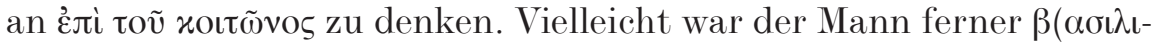
xós) von Melitene und Mesopotamia.

214. Ein Parallelstück Laurent, BZ 33 (1933) 348, Nr. 10.

216. Statt $\mu \varepsilon^{\prime} \gamma[\alpha \varsigma]$ ist wohl $\beta \varepsilon \dot{\sigma} \sigma \tau(\eta \varsigma) \varkappa(\alpha i)$ zu lesen.

217. Vgl. Zacos II 1023.

219. S. DOSeals III 39.49 (Sergios).

220. S. zuletzt DOSeals III 59.6. - 8. Jh.

222. Leider nicht abgebildet; die Rekonstruktion wird DOSeals III 71.4. abgelehnt.

223. Zu einer ev. Verbindung von Optimaton und Dorylaion vgl. DOSeals III 71.22.

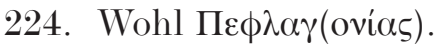

225. ' $\mathrm{H} \mu \lambda \boldsymbol{\varepsilon}^{\prime} \omega v$ wäre belegt: PLP 6708.

228. Das Parallelstück Zacos - Veglery 2621 ist leider auch fragmentiert; vielleicht hieß dieser Stratege von Peloponnesos Darios (Dagios?) und war nur Spatharios.

229. Sicher 9. Jh.

232. Die Ergänzungen werden durch das Parallelstück Zacos II 729 bestätigt.

233. Wie bei einem recht ähnlichen Stück in Birmingham ist als Rangtitel Hypatos anstelle von Bestitor anzunehmen. 


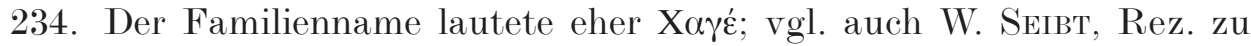
Jordanov, Preslav, BZ 89 (1996) 137 zu Nr. 416-418.

235. Nikephoros Botaneiates war nicht Dux von Strymon und Boleron, sondern von Peloponnesos und Hellas, wie besser erhaltene Parallelstücke zeigen: Auktion Hirsch 175, 23.-26.9.1992, 1567; Auktion Münz Zentrum 94, 13.-15. 5. 1998, 942. Ersteres Kommando ist also aus seinem cursus honorum zu streichen!

236. Dieser Archon war wohl nur $\beta . \varkappa \alpha v \delta ı \delta \tilde{\tau}$ s, nicht Spatharokandidat.

237. Eher ist an einen Strategen zu denken!

238. Vgl. Zacos - Veglery 1691. 8. Jh.

240. Nicht abgebildet, aber vgl. W. SEIBT, Sigillographische Beiträge zur bulgarischen Geschichte. Dobroudja 12 (1995) 226 u. 231, Abb. 3; als Familienname wird dort Proteuon vorgeschlagen.

241. Der Familienname lautet sicher Monokarites, vgl. DOSeals I $43.12 \mathrm{u}$. IV 11.3.

246. Am Ende könnte ein geographischer Terminus stehen; vielleicht Dysis?

248. Am Schluss vermuten wir einen Familiennamen, etwa Achlimanos. Liparites dagegen wird von W. SEIBT, Liparites als „,byzantinischer“ Familienname in der Komnenenzeit, in: Dedicatio (Festschrift M. Lortkipanidze), Tbilisi 2001, 127 abgelehnt.

249. Hieß der Dioiketes vielleicht eher Basileios?

254. Vgl. zuletzt DOSeals III 40.14.

255. Vgl. DOSeals II 64.

256. Vgl. Zacos II 286; DOSeals I 82.8.

261. Zwei Parallelstücke im IFEB.

262. Vgl. DOSeals I 18.36.

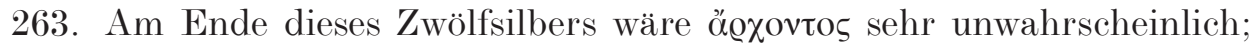

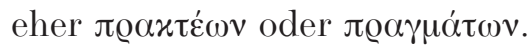

269. 10. Jh.

271. Av. wohl hl. Agathonikos! Der Chartular hieß Konstantin, nicht Nikolaos.

274. Der Mann hieß sicher Theodoros, wie Parallelstücke beweisen (DO 58.106.1081 u. 3003). Auf dem Av. auch das seltenere Tetragramm

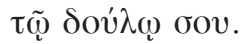

278. Am Ende író

281. Vgl. Zacos - Vegrery 1380. Sollten Pferd und Maultier (?) auf einen

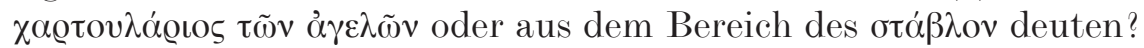

287. Parallelstücke D.O. 58.106.4995; Fogg 287.

288. Der Mann war nicht Dux, denn die Rv.-Legende beginnt mit IWANNI, was auch ein Parallelstück der Sammlung Zacos untermauert. 
294. Vgl. Zacos - Veghery 2389.

295. Der Vatersname war eher Ambros. 7./8. Jh.

296. Parallelstück Seyrig 20; vgl. auch Zacos - Veglery 664.

298. Vgl. Zacos - Veglery 359 u. Auktion Künker (Osnabrück) 20, 30.9.2.10.1991, 1069 .

303. Vgl. Koltsida-Makre 196. 7./8. Jh.

307. S. Corpus II 1027.

309. Eher Theodoros; vgl. auch DOSeals IV zu 11.2.

310. Auf dem Avers Erzengel Michael, nicht hl. Georg.

311. Dieser Gymnos hieß sicher Petros, wie ein Parallelstück der Sammlung Zacos beweist. Mehrere Stufen seiner Karriere sind sigillographisch bezeugt.

312 bis. Vgl. zuletzt W. SEIBт in: Sammlung Köhler-Osbahr (Duisburg) $\mathrm{V} / 4$, Nr. 19.

313. Vgl. Corpus II 876 (nicht stempelidentisch).

317. S. Zacos II 933.

318. Die Rv.-Legende war wohl rein lateinisch: Sergiu magistru mili(tum). Vgl. SeibT, Österreich I zu 197.

319. Zu korrigieren nach Zacos - Veglery 1998. Ioannes war Embolator.

320. Zu ergänzen nach dem Parallelstück Zacos - Veglery 1923. Dieser Meizoteros war ein Georgios Hypatos.

326. Die lateinische Rv.-Legende lautete wohl PROTI-CTU-RUS; vgl. auch Zacos - Veglery 2780. 7. Jh.?

328. Pantherios war nur einfacher Notar, nicht Protonotar.

332. War der Mann Stratege von Charsianon?

337. Vgl. Zacos - Veglery 2355.

340. Ein weiteres Parallelstück zu Corpus II 1084 und entsprechend zu ergänzen bzw. zu korrigieren (Kostomyres).

344. Der Vorname ist sicher unrichtig; vielleicht ICOH.

351. Die Spuren deuten eher in Richtung @oí[ $\varkappa \tau \omega] \varrho \eta \eta^{*}[\dot{\alpha} \mu] \eta^{\prime}$.

354. Sollte Eustratios eher Kumerkiarios von Stratonikeia gewesen sein?

355. Vielleicht mit dem Strategen von Sikelia (DOSeals I 5.30) zu verbinden.

361. Im Gegensatz zu Corpus V 1128 könnte Hamartolos hier tatsächlich als Beiname gemeint sein.

364. Zur Korrektur $\pi \dot{\lambda} \lambda o v \tau \alpha$ s. H. Hunger, Homo Byzantinus. DOP 46 (1992) 124 mit A. 17.

365. Auch Anastasios würde den erhaltenen Buchstaben entsprechen.

366. S. DOSeals II 9.6.

369. Auf Grund der inneren Symmetrie ist für den Bischofssitz primär an Lakedaimonia, Lebedos, Saedron oder Tenedos zu denken. 


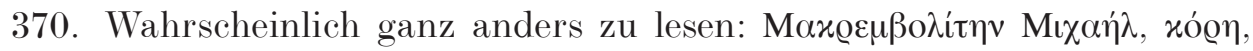
бxغ́лoı५. Vgl. H. Hunger, SBS 5 (1998) 19, Nr. 12 a.

376. Im Corpus V 819 zu Archiepiskopos korrigiert.

377. 2. Hälfte 11. Jh.

378. Im Corpus V 634 schlug Laurent Georgios als Namen vor; vgl. auch V. Grumel, BZ 59 (1966) 395.

379. Vgl. zuletzt DOSeals III 96.1.

380. Im Corpus V 500 wird ein Stufenkreuz auf dem Av. angegeben (so auch Zacos II 150 bis), in der älteren Edition dagegen eine NikolaosBüste.

384. Vgl. Zacos - Veglery 1272 A; 7. Jh.

390. Vgl. Seibt, ByzSl 35 (1974) 82, zu Nr. 1253.

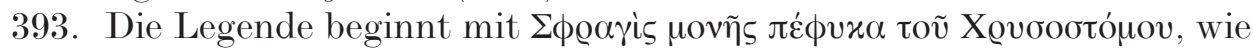
ein Stück der Sammlung Theodoridis lehrt.

395. Abb. auf Taf. 63. Am Ende wäre auch Kardamitu oder Kardamiotu zu überlegen.

396. Wiederholt in Corpus V 1227, mit Recht angezweifelt in Janin, Centres 250 mit Anm. 3.

400. Ein Parallelstück der Sammlung Zacos erlaubt die Korrektur bzw. Ergänzung der Legende: + KE R,, , - IW חPEC-RVTEPO R, -

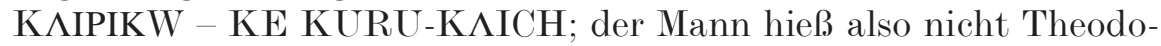
ros!

403. Zacos II 149 bestätigt die Lesung.

408. Schlumberger, Sigillographie 622, las den Namen auf seinem Parallelstück besser: Ariebes. Vgl. auch Müller-Hennig, Bülten 29-30 (1991), 32, Nr. 1. Der Name dürfte zudem Archeologija 1991/1, 45f., Nr. 4 erscheinen.

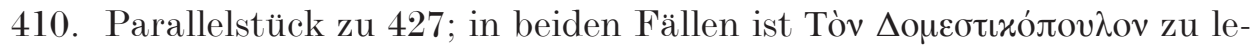
sen, ebenso wie bei Corpus V/2, Nr. 1296 (auch dort falsch gelesen) und Athen $622 \beta$; zu letzterem s. Stavrakos 65.

411. Der Familienname lautet einfach Lu (eher als Ly), ebenso wie beim Typus eines Niketas Lu, Nobellisimos (D.O. 58.106.3467 u. 55.1.3143). Sicher 12. Jh.

412. Vgl. zu SEIBT, Österreich I 162.

415. Konstantin hieß Ardabinos, wie ein (damals nicht als solches erkanntes) Parallelstück zeigt: Seyrig 294.

416. S. SeIBT, Arsakides, JÖB 44 (1994) 351f., Nr. 2.

417. Michael hieß Gelantites, wie auch das Parallelstück D.O. 58.106.1938 zusätzlich absichert.

418. Das Parallelstück Fogg 1383 zeigt, dass das Sigma am Beginn der 2. Zeile steht. 


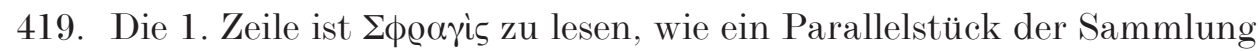
Zacos und das sehr ähnliche Stück D.O. 58.106.4006 unterstreichen.

423. Wahrscheinlich doch Kalypas, wie ein Parallelstück der Sammlung Zacos nahelegt; vgl. auch Kalybas im PLP $10753 \mathrm{f}$.

439. Unter Berücksichtigung des Parallelstückes Fogg 1147 und von Schlumberger, Sig. 629 bzw. Laurent, Bulles métr. 340 (jeweils feh-

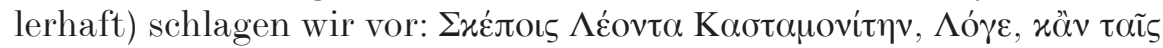

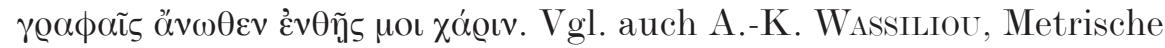
Legenden. Diss. Wien 1998, 1.1.22, Nr. 3.

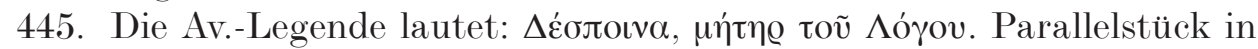
der Sammlung Zacos.

449. Sollte in der zweiten Zeile etwa nur ${ }^{~} \mathrm{P} \omega \mu \alpha v \tilde{\omega}$ zu lesen sein?

454. Parallelstücke in der Sammlung Zacos und in Bulgarien: S. Bilik, Familni imena. Num. i Sfrag.V/2 (1998) 57f., Nr. 12; S. 65, Abb. 13.

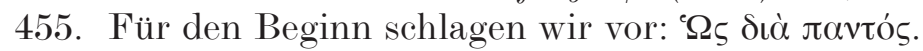

459. Am Ende erwarten wir anstelle des „Familiennamens“ nur oỉxétๆv.

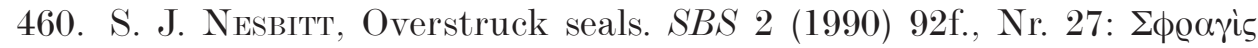

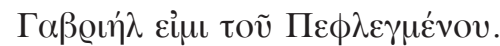

461. Vgl. auch E. Stepanova, Sudak. SBS 6 (1999) 57f., Nr. 22.

464. Parallelstücke D.O. 55.1.2481 und in der Sammlung Zacos. Die Av.-

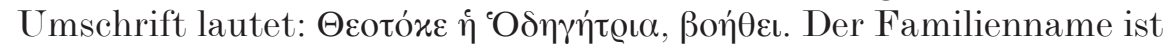
wohl IПOTAMIN(OC) zu lesen.

465. Das zumindest sehr ähnliche Siegel Fogg 1471 hat auf dem Rv. TO. - POA.-NW., weshalb wir den Namen Rodenotes bevorzugen.

466. Saponopulos?

469. Vgl. I. G. Leontiades, Die Tarchaneiotai. Thessaloniki 1998, 115: Am

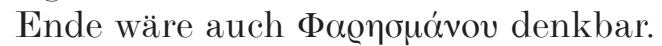

471. Viele Parallelstücke erhalten, vgl. z. B. Jordanov, Preslav 402-403.

474. Parallelstück D.O. 58.106.2938. Av. Büste des hl. Nikolaos. Die Rv.Legende beginnt mit der üblichen Anrufung: + KE R, $\Theta$,.

475. Vgl. auch Seyrig 321.

476. Wenn in der 2. Zeile TW CW $\Delta \mathrm{U}$, stand, folgte wohl IW TPIA (Bischof von Sofia?).

501. Nicht abgebildet, aber wahrscheinlich Parallelstück zu Thierry 165 und einem Siegel im IFEB: Georgios Lachanas.

505. Vgl. Laurent, Bulles métr. 54.

522. Parallelstück zu KoLtsida-MaKre 301 (dort allerdings fehlerhaft).

532. Leider nicht abgebildet. Auf einem möglicherweise sehr nahen Stück

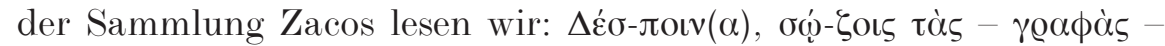
$\mathrm{X} \varrho(v \sigma o) \beta \dot{\varepsilon} \varrho-\gamma(\eta)$.

547. Mehrere Parallelstücke in D.O. 
555. Auf einem recht ähnlichen Siegel der Sammlung Zacos soll die Le-

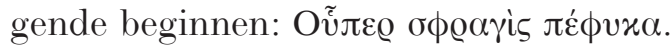

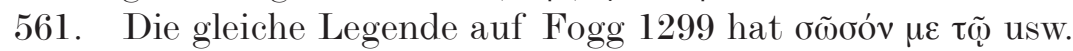

564. Auf einem zumindest sehr nahen Typus (3 Exemplare in D.O.) liest

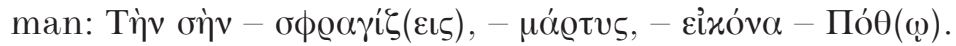

566ff. Bei Monogrammen sind einfache Umzeichnungen oft eine trügerische Arbeitsbasis, daher nur wenige Bemerkungen:

570. Vgl Zacos - Vegleri 295.

573. Das Rv.-Monogramm in dieser Form würde eher Eủooxínov, allenfalls $\Delta \varepsilon x i \mu o v$ ergeben.

580. Theodosios käme sogar noch eher in Frage.

598. Vgl. Zacos - Veglery 1193.

599. Vgl. Zacos - Veglery 1201.

602. Parallelstück Zacos - Veglery 1618.

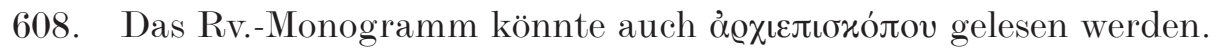

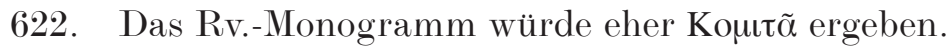

628. Rv.-Monogramm Eủxa@íotov? Vgl. Zacos - Vegler 728.

691. Vgl. Zacos - Veglery 2996 bis. 
